MEIRELLES, L.M.A.

REF-ISSN1808-0804 Vol.XI (4),06-26, 2014.

\title{
ESTABILIDADE DE MEDICAMENTOS: ESTADO DA ARTE.
}

DRUG STABILITY: STATE OF THE ART.

ESTABILIDAD DE MEDICAMENTOS: ESTADO DEL ARTE.

Lyghia Maria Araújo Meirelles ${ }^{1}$

Faculdade Santo Agostinho - Curso de Farmácia

Av. Valter Alencar 665 - São Pedro Teresina - PI - Cep: 64.019-625 Fone: (86) 3215-8700

Email: lyghia@ymail.com

Submetido em 02/07/2014, Aceito em 29/10/2014

RESUMO: A estabilidade de medicamentos é a extensão na qual um produto mantém suas características dentro de critérios de aceitação ao longo do tempo. Para determinação da estabilidade de um medicamento são avaliados fatores interferentes de caráter extrínseco, como temperatura, umidade ou luz, ou intrínsecos, como as propriedades físico-químicas do fármaco e excipientes, o processo de obtenção e a forma farmacêutica. Embora diversos países tenham sua própria norma para realização dos estudos de estabilidade, a Conferência Internacional de Harmonização e a Organização Mundial de Saúde têm direcionado esforços no sentido de uniformizar a condução dos estudos de estabilidade, pois é crescente a transferência de tecnologias e produtos entre países. Esta padronização se faz necessária, pois o prazo de validade e as condições de armazenagem do medicamento são determinados conforme a zona climática da região na qual será comercializado. Para contornar potenciais interferentes, podem ser adotadas estratégias, como formulações extemporâneas, uso de excipientes funcionais, e condições de armazenamento e acondicionamento diferenciadas. Portanto, a execução de estudos de estabilidade previamente à disposição do produto no mercado pode evitar descobertas tardias de ineficácia terapêutica e toxicidade. 


\section{i) Revista Eletrônica de Farmácia}

MEIRELLES, L.M.A.

REF-ISSN1808-0804 Vol.XI (4),06-26, 2014.

PALAVRAS-ChAVE: Formas de dosagem, Estabilidade de medicamentos, Prazo de validade.

ABSTRACT: The stability of drugs is the extent to which a product retains its characteristics within acceptance criteria over time. To determine the stability of a drug interfering factors extrinsic character, such as temperature, humidity or light, or intrinsic, such as the physicochemical properties of the drug and excipients, the process of getting and the dosage form are evaluated. Although many countries have their own standards for performing the stability studies, the International Conference on Harmonization and the World Health Organization have directed efforts to standardize the conduct of stability studies, since it is increasing the transfer of technologies and products between countries. This standardization is necessary because the shelf life and storage conditions of the product are determined according to the climate zone wherein will be marketed. To circumvent potential interfering strategies can be adopted as extemporaneous formulations, use of functional excipients and storage and packaging conditions different. Therefore, the implementation of stability studies previously available product on the market can avoid late discovery of therapeutic inefficacy and toxicity.

KEYWORDS: Dosage forms, Drug stability, Date of Validity of Products.

RESUMEN: La estabilidad de los medicamentos es el grado en que un producto conserva sus características dentro de los criterios de aceptación en el tiempo. Para determinar la estabilidad de un medicamento factores interfierentes de carácter extrínseco, tales como la temperatura, la humedad o la luz, o intrínseco, tales como las propiedades fisicoquímicas del fármaco y los excipientes, el proceso de obtención y la forma de dosificación se evalúan. Aunque muchos países tengan sus propias normas para la realización de los estudios de estabilidad, la Conferencia Internacional sobre Armonización y la Organización Mundial de la Salud ha dirigido esfuerzos para normalizar la conducta de los estudios de estabilidad, ya que está aumentando la transferencia de tecnologías y productos entre países. Esta estandarización es necesaria porque las condiciones de vida y de almacenamiento útil del producto se determinan de acuerdo con la zona climática de la región en 
MEIRELLES, L.M.A.

REF-ISSN1808-0804 Vol.XI (4),06-26, 2014.

que se comercializará. Para eludir los interferentes potenciales se puede adoptar estrategias como formulaciones extemporáneas, el uso de excipientes funcionales y condiciones diferentes de almacenamiento y envase. Por lo tanto, la implementación de estudios de estabilidad de productos previamente a su disposición en el mercado puede evitar el descubrimiento tardío de ineficacia terapéutica y la toxicidad.

PALABRAS CLAVE: Formas de Dosificación; Estabilidad de Medicamentos; Fecha de Caducidad de Productos

\section{INTRODUÇÃO}

Antes de comercializar um novo fármaco ou medicamento a indústria deve realizar uma série de ensaios para avaliar sua conformidade com as especificações físico-químicas e microbiológicas sob condições compatíveis com a zona climática da região geográfica onde o produto será distribuído. A fim de regulamentar a variabilidade dos parâmetros de condução deste estudo algumas mudanças ocorreram ao longo do tempo ${ }^{[1-2]}$.

Em 1892 os Estados Unidos constituíram o primeiro conjunto de normas para o controle de fármacos, entretanto, somente em 1936 a Farmacopeia Portuguesa estabeleceu que diversas formas farmacêuticas fossem preparadas somente no momento da administração e determinou prazo de eficácia para soros e vacinas ${ }^{[2]}$.

$\mathrm{Na}$ década de 50, concomitantemente aos acidentes que incentivaram a farmacovigilância, estabeleceram-se as primeiras alternativas para previsão do prazo de validade de medicamentos. Com o desenvolvimento científico e tecnológico, os métodos empíricos foram deixados de lado e ensaios com maior embasamento matemático passaram a ser adotados, ao passo que os órgãos fiscalizadores iniciaram a publicação de normas e guias para orientar o setor regulado. A OMS iniciou sua colaboração a partir de 1988, quando publicou o Guidelines on Stability Testing for Well Stablished Drug on Conventional Dosage Forms e em 1993 o ICH publicou o Guideline on Stability Testing (Q1A). $\mathrm{O}$ estudo de estabilidade foi um dos primeiros 
MEIRELLES, L.M.A.

REF-ISSN1808-0804 Vol.XI (4),06-26, 2014.

tópicos que foi adotada a padronização ${ }^{[3-4]}$.

Estabilidade é a extensão na qual um produto mantém, dentro dos limites especificados e por todo o seu prazo de validade, as mesmas características que possuía no momento de sua fabricação. Este é um atributo crítico de qualidade, regulamentado em âmbito nacional sob a forma do Guia para realização de estudos de estabilidade (RE no 01/2005), além de compor as Boas Práticas de Fabricação (RDC no 17/2010). O objetivo inicial da adoção deste tipo de estudo foi definir o prazo de validade de medicamentos, no entanto, um enfoque maior tem sido dado à segurança dos produtos farmacêuticos, através da determinação e quantificação de produtos de degradação. Preocupação esta confirmada pela Consulta Pública no 11/2012, que trata do estabelecimento de parâmetros para a notificação, identificação e qualificação de produtos de degradação em medicamentos ${ }^{[3,5,6]}$.
Assim, o presente trabalho visa apresentar uma revisão sobre os testes de estabilidade e sua importância durante o delineamento de um medicamento, expor as principais técnicas para avaliação da compatibilidade fármaco-excipiente, elencar as instabilidades mais comuns em produtos farmacêuticos, além de tratar da condução de estudos de estabilidade forçada para obtenção e caracterização de produtos de degradação.

\section{Tipos de estabilidade}

A estabilidade de produtos farmacêuticos é afetada por fatores extrínsecos ou ambientais, como temperatura, umidade e luz, e intrínsecos ou relacionados ao produto, como propriedades físicoquímicas dos constituintes, forma farmacêutica, processo de fabricação e materiais de embalagem. As variáveis avaliadas nos estudos de estabilidade podem ser classificadas conforme a Figura $1^{[6]}$. 
MEIRELLES, L.M.A.

REF-ISSN1808-0804 Vol.XI (4),06-26, 2014.

Figura 1. Tipos de Estabilidade.

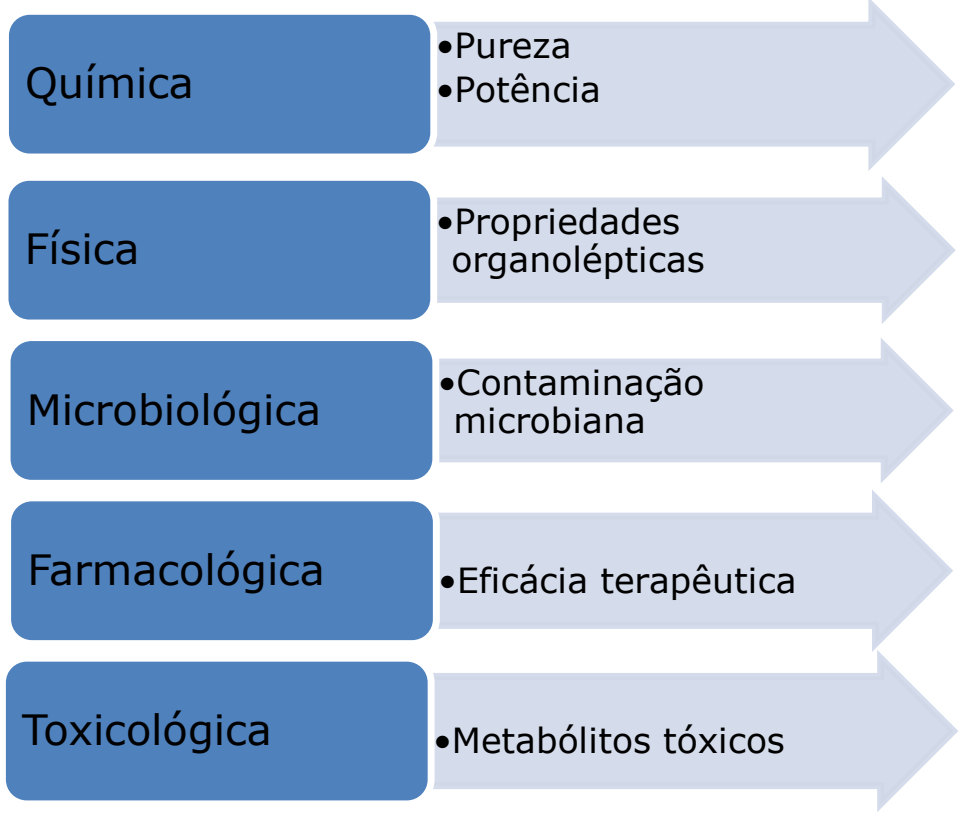

A estabilidade química provavelmente é o aspecto mais importante da estabilidade farmacêutica, pois determina as incompatibilidades fármaco-excipiente na formulação e permite selecionar as condições de armazenamento e acondicionamento compatíveis com o produto. A manutenção das características físicas está relacionada aos aspectos intrínsecos do fármaco ou forma farmacêutica, podendo ter influência do material de embalagem devido sua permeabilidade. Comprimidos podem tornar-se mais friáveis ao longo tempo, da mesma forma que uma emulsão poderá sofrer coalescência, com quebra da emulsão, quando submetida a condições ambientais adversas ${ }^{[6,8]}$.

A conservação da formulação contra a contaminação microbiológica pode ser atingida com a adoção de Boas Práticas de Fabricação, um rigoroso controle ambiental $\mathrm{e}$ emprego racional de agentes conservantes, de modo que os mesmos não mascarem o crescimento microbiano advindo do processo de 
MEIRELLES, L.M.A.

REF-ISSN1808-0804 Vol.XI (4),06-26, 2014.

fabricação, ou sejam nocivos ao paciente ${ }^{[9]}$.

As propriedades terapêuticas e toxicológicas são cruciais durante o screening de moléculas potencialmente bioativas. Os aspectos farmacocinéticos e farmacodinâmicos são essenciais para definição da indicação terapêutica e posologia, enquanto os toxicológicos avaliam a ação nociva de impurezas e produtos de degradação no organismo ${ }^{[6]}$.

\section{Testes de estabilidade}

O estudo de estabilidade é uma etapa fundamental para garantir a qualidade, segurança e eficácia de um medicamento. Durante o estudo de pré-formulação parâmetros de estabilidade são avaliados, conforme sequência descrita na Figura 2. A partir dos resultados obtêm-se evidências sobre o comportamento do medicamento, sob a influência de fatores extrínsecos e intrínsecos, estabelecendo o prazo de validade e as condições de armazenamento e acondicionamento adequadas ${ }^{[1]}$.

Figura 2. Considerações sobre estabilidade no desenvolvimento de um medicamento.

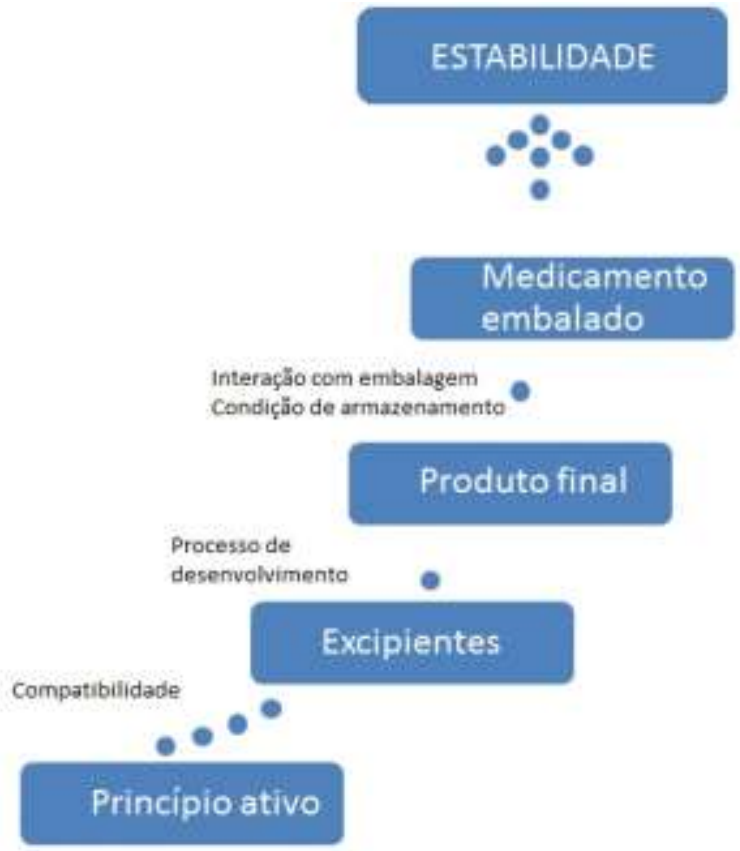

Os estudos de estabilidade produtos farmacêuticos serão devem garantir que a identidade, preservadas durante todo o seu prazo potência, pureza e inocuidade dos de validade. Tais estudos são 
MEIRELLES, L.M.A.

REF-ISSN1808-0804 Vol.XI (4),06-26, 2014.

classificados em acelerado, de longa duração e de acompanhamento. Estes estudos devem ser realizados de acordo com as condições climáticas do país onde o registro foi solicitado, considerando a zona climática a qual ele pertença. O Brasil é classificado como zona climática IV, país de clima quente e úmido ${ }^{[10]}$.

O estudo acelerado induz o aumento da velocidade de degradação química e/ou alterações físicas no produto farmacêutico pela utilização de condições drásticas de armazenamento, com o propósito de monitorar as reações de degradação e estimar o prazo de validade, durante um período de seis meses ${ }^{[7]}$.

O estudo de longa duração é realizado em condições normais de armazenamento, como o objetivo de confirmar dados obtidos no estudo acelerado e determinação do prazo de validade do produto farmacêutico. As análises são realizadas inicialmente durante 24 meses, porém é facultado ao o fabricante prosseguir os estudos a fim de prolongar o prazo de validade, mediante a manutenção dos limites de aceitação ${ }^{[11]}$.

Com os resultados obtidos nos estudos de estabilidade acelerada e de longa duração, é possível avaliar qualitativa e quantitativamente as alterações químicas e físicas às quais o produto está sujeito, permitindo estabelecer também a compatibilidade entre $o$ produto e a embalagem primária $^{[7,11]}$.

O estudo de estabilidade de acompanhamento é realizado após concessão do registro ou notificação do produto, e tem por objetivo verificar a manutenção das suas características, conforme os resultados obtidos nos estudos preliminares. Desta forma, assegurase que um controle de mudanças efetivo é adotado pela empresa e seu impacto sobre o produto final avaliado periodicamente ${ }^{[7]}$.

\section{Compatibilidade excipiente}

fármaco-

Via de regra, um medicamento é composto por um ou mais fármacos associados a excipientes, os quais atuam como auxiliares na fabricação ou performance do produto final. A seleção dos excipientes é vital para o desenvolvimento de um medicamento, pois é sabido que embora não possuam atividade farmacológica, sua composição qualitativa e quantitativa na fórmula influencia o sistema de liberação e a 


\section{- Revista Eletrônica de Farmácia}

MEIRELLES, L.M.A.

REF-ISSN1808-0804 Vol.XI (4),06-26, 2014.

biodisponibilidade do princípio ativo ${ }^{[12-}$ 13].

O estudo de pré-formulação é essencial para conhecer as propriedades fundamentais $\mathrm{e}$ derivadas do fármaco e seus excipientes, constituindo uma fase de transição entre as etapas química e tecnológica. Nesta fase é possível obter informações sobre a estabilidade do fármaco e da formulação e a compatibilidade entre princípios ativos e excipientes. Pois, embora sejam caracterizados como substâncias inertes, os adjuvantes possuem grupos funcionais que favorecem a interação com outras substâncias, tanto no estado sólido como em solução ${ }^{[14-18]}$.

A presença de traços de impurezas dos excipientes pode afetar a qualidade, segurança e eficácia de medicamentos devido a formação de produtos de degradação ativos ou redução da potência do insumo ativo, especialmente nos casos em que o fármaco possui alta potência e baixa dosagem e a relação excipiente/fármaco é alta. Portanto, para o delineamento de uma fórmula robusta a escolha dos excipientes deve considerar tanto a funcionalidade quanto a compatibilidade química das substâncias. Compatibilidade que, ao ser avaliada, pode levar a economia em termos de recursos e tempo, além de evitar problemas tardios de estabilidade ${ }^{[19-21]}$.

A análise térmica, empregada na caracterização, estudos de pureza, determinação de polimorfismo e cinética de degradação térmica de fármacos, também permite a visualização das interações entre 0 ativo e demais componentes da formulação. Algumas técnicas envolvento análise térmica têm sido utilizadas como ferramenta para a avaliação rápida de compatibilidade em estudos de pré-formulação, embora não substituam completamente o programa clássico de estabilidade ${ }^{[21-23]}$.

As técnicas mais empregadas são a termogravimetria (TG) e a calorimetria exploratória diferencial (DSC). O sistema simultâneo de TG / DSC é uma ferramenta muito útil para a investigação de compostos orgânicos, pois, em uma única análise, determina a perda de massa e fluxo de calor nas amostras. Transformações que ocorrem, mesmo com pequena oscilação de massa (reações químicas, vaporização de decomposição e processos oxidativos), podem ser diferenciadas 
MEIRELLES, L.M.A.

REF-ISSN1808-0804 Vol.XI (4),06-26, 2014.

daquelas que ocorrem sem variação aparente (fusão, transição vítrea, alterações polimórficas) ${ }^{[24-26]}$.

As curvas obtidas no TG dão indício de perdas de massa associadas à evaporação de água ou solventes, no caso de hidratos e solvatos, seguidos de perda associada à decomposição do fármaco. Ao confrontar estes resultados com a curva do DSC confirmam-se os eventos observados no TG, representados por eventos físicos $\mathrm{e}$ químicos decorrentes da variação de energia, entalpia. A interconversão de polimorfos, por exemplo, pode representar um problema durante 0 processamento e armazenamento de insumos que possue formas instáveis, para tanto deve-se assegurar condições para que a forma polimórfica ideal mantenha-se inalterada na formulação, atendendo requisitos de solubilidade e estabilidade $\mathrm{e}^{[27-28]}$.

O estudo de compatibilidade deve avaliar isoladamente o fármaco e excipientes da formulação e sistemas binários do fármacoexcipiente. Sob temperatura inferior ao início de decomposição do fármaco, espera-se que haja uma sobreposição dos perfis térmicos individuais dos insumos analisados, mantendo-se nítido o evento referente à fusão do princípio ativo. Alterações na faixa de fusão, forma ou área dos picos, bem como, aparecimento ou desaparecimento de eventos térmicos nos sistemas binários podem indicar interações químicas ${ }^{[24,27]}$.

Embora haja algumas vantagens no TG / DSC, como a necessidade de pequena quantidade de amostra, a rapidez e versatilidade, há também algumas limitações. Situações em que o fármaco é amorfo ou solúvel no excipiente podem provocar uma perda de entalpia que deve ser interpretada com cautela, para tanto é preferível a associação de outras técnicas, como cromatografia ou difração de raios $X^{[27,29,30,31]}$.

\section{Estudos de degradação}

Para auxiliar na predição da estabilidade do fármaco os estudos de degradação são realizados mediante exposição do medicamento a condições reais de estresse, induzindo redução do teor do ativo e aumento da quantidade de produtos de degradação. A degradação da amostra é induzida através do aumento da temperatura e / ou umidade, da exposição a condições variadas de pH, luz intensa (fotoestabilidade) e 


\section{- Revista Eletrônica}

MEIRELLES, L.M.A.

REF-ISSN1808-0804 Vol.XI (4),06-26, 2014.

agentes oxidantes ou metálicos.

Métodos indicativos de estabilidade devem ser desenvolvidos para identificar e quantificar as impurezas ${ }^{[1,5,21]}$.

A Consulta Pública no 11/2012 determina que 0 teste deva proporcionar uma degradação de 10 a $30 \%$, cujo limite de detecção e quantificação é atribuído de acordo com a dose diária máxima. As condições ambientais para conservação da amostra submetida ao estudo de degradação forçada são estabelecidas no Quadro 1, conforme Huynh-Ba ${ }^{[1]}$. Para realização do estudo utilizam-se câmaras climáticas, as quais devem conter os produtos em sua embalagem primária sob as condições especificadas. É relevante a realização de um ensaio controle com o placebo para verificar se a origem dos produtos de degradação é fármaco-dependente ou não ${ }^{[5]}$.

Quadro 1. Condições de estresse recomendadas para medicamentos.

\begin{tabular}{cc}
\hline Parâmetros & Condições \\
\hline Uemperatura & $70^{\circ} \mathrm{C}$ \\
\hline Umidade & $75 \%$ UR \\
\hline Ootoestabilidade & $\begin{array}{c}\text { Luz ultravioleta ou } \\
\text { fluorescente }\end{array}$ \\
\hline Hidrólise ácida & $\mathrm{H}_{2} \mathrm{O}_{2} 0,3 \%$ \\
\hline Hidrólise básica & $\mathrm{HCl} 0,1 \mathrm{M}$ \\
\hline
\end{tabular}

Alguns dos fatores que levam às condições de estresse, no entanto, podem afetar o produto antes mesmo de chegar ao fim do processo fabril. Portanto é necessário um ambiente controlado para a manipulação de substâncias instáveis quando expostas à luz, altas temperaturas ou umidade relativa do ar elevada[21].

A decomposição de um fármaco normalmente constitui um processo exotérmico, ou seja, um processo que 
MEIRELLES, L.M.A.

REF-ISSN1808-0804 Vol.XI (4),06-26, 2014.

é acompanhado por uma evolução de calor. 0 deslocamento, 0 aparecimento ou o desaparecimento dos picos endotérmicos ou exotérmicos e a mudança de outros valores entálpicos em curvas térmicas obtidas através de DSC, podem ser indicativos da formação de subprodutos. Assim como o desaparecimento ou redução de bandas de absorção, junto ao aparecimento de novas bandas, em espectros no FT-IR é evidência de interação entre o fármaco e os excipientes em estudo ${ }^{[30,32,33]}$.

Em estudos mais detalhados, os produtos de degradação podem ser identificados mediante o acoplamento de diferentes técnicas. A separação fornecida por um sistema cromatográfico, como o HPLC, combinado a uma técnica quantitativa, sensível e específica, possibilita a detecção de impurezas em misturas complexas, tais como formulações, oferecendo maiores informações sobre a via de degradação. Os subprodutos detectados podem ser isolados e caracterizados, através de análise comparativa com padrões de referência destes ${ }^{[31,34]}$.

Os compêndios oficiais reportam os principais produtos de decomposição dos insumos farmacêuticos e os disponibilizam sob a forma de padrões com elevada pureza. Os métodos de análise, mesmo quando farmacopeicos, devem ser validados. E a pureza cromatográfica da amostra deve ser realizada no início e no final do estudo. Dentre as técnicas para detecção e quantificação de tais compostos as mais utilizadas são a cromatografia líquida de alta eficiência, espectrofotometria no ultravioleta-visível, espectroscopia na região do infravermelho, espectrometria de massa e análise térmica ${ }^{[5,25]}$.

\section{Cinética de reação}

Cinética é o estudo da velocidade na qual os processos ocorrem, objetivando fornecer informações para a compreensão dos mecanismos responsáveis pelas transformações e a previsão quantitativa desta alteração após um dado intervalo de tempo. As transformações podem ser químicas ou físicas, as quais permitem uma abordagem racional sobre a estabilidade e a produção de medicamentos, e a predição do prazo 
MEIRELLES, L.M.A.

REF-ISSN1808-0804 Vol.XI (4),06-26, 2014.

de validade a partir de modelos matemáticos ${ }^{[3,26,35]}$.

A análise da cinética de decomposição deverá descrever de modo adequado a ordem de reação e os parâmetros de Arrhenius. As reações de degradação de produtos farmacêuticos ocorrem sob condições definidas, portanto é preciso conhecer a ordem e a velocidade de reação para determinar a concentração do fármaco em função do tempo. Em geral, estas reações podem ser de ordem zero (quando a velocidade independe das concentrações dos reagentes) e de primeira ordem (quando a velocidade de reação é proporcional à concentração de um reagente) e, mais raramente, de segunda ordem (quando a velocidade de reação é proporcional ao produto das concentrações de dois reagentes) $)^{[6,21,26,32]}$.

As técnicas termoanalíticas podem ser utilizadas com o intuito de definir os parâmetros cinéticos em estudos de reação no estado sólido, como por exemplo, a energia de ativação (Ea), o fator frequência $(A)$ e a ordem de reação $(n)$. Então, tornase possível avaliar variações de propriedades da amostra quando esta é colocada em contato com adjuvantes, submetida à atmosfera oxidante ou umidade. Porém, qualquer metodologia analítica capaz de medir o consumo de reagentes ou formação de produtos pode ter seus dados convertidos em gráficos da concentração dos produtos de degradação em função do tempo ou temperatura ${ }^{[36-40]}$.

O método convencional empregado no estudo cinético de fármacos, dito método isotérmico, é o que utiliza um aquecimento constante, próximo da temperatura de decomposição da amostra. No método isotérmico o cálculo da energia de ativação é baseado na equação de Arrhenius. O método descrito por Ozawa baseia-se na variação da temperatura, por isso é conhecido por não-isotérmico ou dinâmico, e é bastante utilizado em estudos de estabilidade térmica. Este modelo permite encontrar os parâmetros cinéticos a partir da integração da equação de Arrhenius ${ }^{[39-41]}$.

\section{Estabilidade do produto final}

Para o registro, e consequente comercialização de um produto, é necessário que a empresa responsável pelo registro tenha concluído os estudos de estabilidade acelerada e os ensaios de longa duração estejam em 
MEIRELLES, L.M.A.

REF-ISSN1808-0804 Vol.XI (4),06-26, 2014.

execução. 0 estudo de

produto e alterações em etapas acompanhamento é uma maneira de assegurar a manutenção dos parâmetros de qualidade dos produtos enquanto forem fabricados pela empresa, verificando a manutenção das características mediante um controle de mudanças. Portanto, com o início do processo de fabricação, o produto não poderá sofrer variações sem a avaliação do impacto das mudanças na estabilidade do medicamento. São consideradas alterações significativas alterações na composição quali e/ou quantitativa do críticas do processo e do material de embalagem ${ }^{[7,42]}$.

Para contornar potenciais interferentes detectados no estudo de pré-formulação, é possível adotar uma série de estratégias, ajustando desde a composição do medicamento até as condições de armazenamento e acondicionamento. O Quadro 2 demonstra alguns atributos de formas farmacêuticas que podem sofrer alterações durante a armazenagem, e que influenciam a segurança, qualidade e eficácia do produto ${ }^{[21,35]}$.

Quadro 2. Parâmetros indicativos de estabilidade segundo a forma farmacêutica.

\begin{tabular}{|c|c|}
\hline $\begin{array}{c}\text { Formas } \\
\text { Farmacêuticas }\end{array}$ & \multicolumn{1}{|c|}{ Parâmetros } \\
\hline Sólidas & $\begin{array}{c}\text { Aspecto, dureza, dissolução, teor, produtos } \\
\text { de degradação, limites microbianos }\end{array}$ \\
\hline Líquidas \& & $\begin{array}{l}\text { Aspecto, pH, sedimentação pós agitação } \\
\text { com baspensões), perda de peso (produtos } \\
\text { (emulsões e cremes), claridade (soluções), } \\
\text { teor, produtos de degradação, limites } \\
\text { microbianos }\end{array}$ \\
\hline
\end{tabular}

Preparações farmacêuticas sujeitas à hidrólise devem ter a água reduzida ou eliminada do sistema. O uso de revestimento em formas farmacêuticas sólidas protege o medicamento da luminosidade e umidade ambiental e o acondicionamento em recipiente 
MEIRELLES, L.M.A.

REF-ISSN1808-0804 Vol.XI (4),06-26, 2014.

hermeticamente fechado minimiza a oxidação. Em formulações líquidas a água pode ser reduzida ou substituída por outros solventes, o fármaco hidrolisável poderá ser suspenso em veículo não-aquoso ou utilizado extemporaneamente. A refrigeração e a manutenção do $\mathrm{pH}$ dentre de uma faixa ótima geralmente garantem uma melhor estabilidade do produto $^{[6,43]}$.

A oxidação de princípios ativos ou adjuvantes geralmente é acompanhada de alteração na cor do produto, ou ainda em precipitação e mudança do odor. O processo oxidativo pode ser evitado através do uso de antioxidantes, os quais fornecerão elétrons ou prótons lábeis captados por radicais livres promotores de instabilidade. Para tanto, é preciso que o adjuvante seja mais prontamente oxidado que 0 fármaco. São mais suscetíveis à oxidação moléculas que contenham fenóis, aminas aromáticas, aldeídos, éteres e compostos alifáticos não saturados. Diversos antioxidantes estão disponíveis no mercado, por exemplo, o metabissulfito de sódio, ácido hipofosfórico, ácido ascórbico e alfa-tocoferol ${ }^{[6,35]}$.

A oxidação e, em certo grau, a hidrólise são muitas vezes catalisadas pela luz, que pode ter efeitos sobre 0 fármaco, bem como o produto final ou material de embalagem. Geralmente a radiação ultravioleta, seja de fonte natural ou artificial, causa fotodegradação de fármacos, tais como riboflavina, nifedipino e fenotiazinas. Embora muitos fármacos sejam fotossensíveis, as consequências práticas podem não ser necessariamente as mesmas para todos os compostos. Ou seja, precauções distintas serão exigidas no manuseio desses produtos, como o uso de uma embalagem opaca ou âmbar, revestimentos ou embalagens secundárias $^{[35,44]}$.

Além dos adjuvantes convencionais, técnicas de produção e embalagens diferenciadas têm sido ferramentas úteis para assegurar a estabilidade de medicamentos. Os carreadores empregados em sistemas de liberação e complexos de inclusão conferem maior estabilidade a fármacos com propriedades limitantes. As ciclodextrinas (CDs), por exemplo, têm importância na otimização de formulações de fármacos pouco solúveis em água, tanto pelo efeito solubilizante, quanto estabilizante, que está diretamente relacionado com a inclusão $d a$ molécula na cavidade da $C D$ e, 
MEIRELLES, L.M.A.

REF-ISSN1808-0804 Vol.XI (4),06-26, 2014.

consequentemente, dos grupos químicos suscetíveis a reagir ${ }^{[14,45,46]}$.

Da mesma forma, polímeros produzem um sistema matricial para liberação modificada de fármacos. Sistemas de revestimento entérico permitem que fármacos ácido-lábeis atinjam 0 intestino de forma inalterada. Materiais poliméricos biocompatíveis como podem ser utilizados como membranas ou matrizes nas quais o ingrediente ativo é disperso ou dissolvido, por exemplo derivados da celulose, argilas e carbômeros $^{[47-49]}$.

\section{CONSIDERAÇÕES FINAIS}

A estabilidade é um tema relativamente recente no âmbito da indústria farmacêutica e as agências regulamentadoras tendem a aumentar as exigências, de modo que os processos e especificações tornem-se mais definidos e condizentes com as tecnologias adotadas pelo setor regulado. Apenas com um maior rigor técnico será possível detectar e solucionar problemas farmacotécnicos e analíticos até então desconhecidos. Cabe, então, uma maior colaboração para caracterização e síntese de produtos de degradação, fornecimento de um número mais significativo de seus padrões de referência, desenvolvimento de métodos analíticos indicativos de estabilidade, além de maior esclarecimento quanto à condução destes estudos e sua interpretação. Desta forma, o medicamento terá garantia de alcançar o paciente em condições adequadas de uso, obtendo a ação terapêutica esperada durante o período previsto.

\section{REFERÊNCIAS BIBLIOGRÁFICAS}

1. Huynh-Ba K, editor. Handbook of Stability Testing in Pharmaceutical Development. 1st ed. Newark: Springer; 2009.

2. Prista LN, Alves AC, Morgado R. Técnica farmacêutica e farmácia galênica. 3rd ed. Lisboa: Fundação Galouste Guldenkian; 1995. 


\section{i) Revista Eletrônica}

MEIRELLES, L.M.A.

REF-ISSN1808-0804 Vol.XI (4),06-26, 2014.

3. Leite EG. Estabilidade: importante parâmetro para avaliar a qualidade, segurança e eficácia de fármacos e medicamentos. [thesis]. Porto Alegre: Faculdade de Farmácia/Universidade Federal do Rio Grande do Sul; 2006. 178 p.

4. Carvalho JP, Santos AS, de Sá AS, Teixeira TS, Nogueira MS. Estabilidade de medicamentos no âmbito da farmacovigilância. Fármacos e Medicamentos. 2005; 34(6):22-27.

5. Ministério da Saúde. Agência Nacional de Vigilância Sanitária. Consulta Pública CP n 11, de 23 de janeiro de 2012. Estabelece parâmetros para a notificação, identificação e qualificação de produtos de degradação em medicamentos com princípios ativos sintéticos e semi-sintéticos, classificados como novos, genéricos e similares, e dá outras providências. Brasília (Brasil): Ministério da Saúde; 2012.

6. Allen-Jr LV, Popovich NG, Ansel HC. Formas farmacêuticas e sistemas de liberação de fármacos. 8 ed. Porto Alegre: Artmed; 2007.

7. Ministério da Saúde. Agência Nacional de Vigilância Sanitária. Resolução $n^{\circ} 01$, de 29 de julho de 2005 - Guia para realização de estudos de estabilidade. Brasília (Brasil): Ministério da Saúde; 2005.

8. Laszcz M, Trzcinka K, Kubiszewski M, Kosmacinska B, Glice M. Stability studies and structural characterization of pramipexole. J Pharm Biomed Anal. 2010;53(4): 1033-1036.

9. Pinto TJA, Kaneko TM, Pinto AF. Controle Biológico de Qualidade de Produtos Farmacêuticos, Correlatos e Cosméticos. 3rd ed. São Paulo: Atheneu; 2010.

10. Gil ES. Controle de qualidade físico-químico de medicamentos. 3rd ed. São Paulo: Pharmabooks; 2010. 


\section{) Revista Eletrônica}

MEIRELLES, L.M.A.

REF-ISSN1808-0804 Vol.XI (4),06-26, 2014.

11. International Conference on Harmonisation [Internet]. Guidance for industry Q1A(R2) stability testing of new drug substances and products; 2003 [cited 2013 out 24]. Available from:

http://www.ich.org/fileadmin/Public_Web_Site/ICH_Products/Guidelines/Quality/Q1 A_R2/Step4/Q1A_R2_Guideline.pdf.

12. Ferreira TF, Mourão ASR, Ribeiro LAL, Freitas MB. Estudo comparativo da influência dos excipientes na qualidade de hidroclorotiazida $25 \mathrm{mg}$ em medicamentos referência e genéricos. Rev Ciênc Farm Básica Apl. 2013;34(1):6368.

13. Kushner-IV J, Langdon BA, Hiller JI, Carlson GT. Examining the impact of excipient material property variation on drug product quality attributes: a qualityby-design study for a roller compacted, immediate release tablet. J Pharm Sci. $2011 ; 100(6): 2222-2239$.

14. Barreto LCLS, Cunha-Filho MSS. Ciclodextrina: Importante Excipiente Farmacêutico Funcional. Lat Am J Pharm. 2008;27(4):629-636.

15. Yu H, Cornett $\mathrm{C}$, Jesper L, Hansen SH. Reaction between drug substances and pharmaceutical excipients: Formation of esters between cetirizine and polyols. J Pharm Biomed Anal. 2010;53(3):745-750.

16. Paula IC. Proposta de um modelo de referência para o processo de desenvolvimento de produtos farmacêuticos. [thesis] Porto Alegre: Escola de Engenharia/Universidade Federal do Rio Grande do Sul; 2004. 314 p.

17. Araújo AAS. Análise térmica e determinação dos parâmetros cinéticos de preparações farmacêuticas e novas especialidades de zidovudina. [dissertation]. São Paulo: Faculdade de Ciências Farmacêuticas/Universidade de São Paulo; 2003. $241 \mathrm{p}$. 
MEIRELLES, L.M.A.

REF-ISSN1808-0804 Vol.XI (4),06-26, 2014.

18. Souza FS, Macedo RO, Veras JWE. Studies of cimetidine pre-formulated and tablets for TG ans DSC coupled to the photovisual system. Thermochim Acta. 2002; 392-393:99-106.

19. Wu Y, Levons J, Narang AS, Raghavan K, Rao VM. Reactive impurities in excipients: profiling, identification and mitigation of drug-excipient incompatibility. AAPS PharmSciTech. Dec 2011;12(4):1248-1263.

20. Dousa M, Gibala P, Havlicek J, Placek L, Tkadlecova M, Brichac J. Drug-excipient compatibility testing-Identification and characterization of degradation products of phenylephrine in several pharmaceutical formulations against the common cold. J Pharm Biomed Anal. 2011;55(5):949-956.

21. Qiu Y, Chen Y, Zhang GGZ, Liu L, Porter W. Developing solid oral dosage forms: Pharmaceutical Theory and Pratices. 1st ed. New York: Elsevier; 2009.

22. Soares-Sobrinho JL, Soares MFLR, Lopes PQ, Correia LP, de Souza FB, Macêdo RO et al. A preformulation study of a new medicine for chagas disease treatment: physicochemical characterization, thermal stability, and compatibility of benznidazole. AAPS PharmSciTech. 2010;11(3):1391-1396.

23. Silva KER, Alves LDS, Soares MFR, Passos RCS, Faria AR, Rolim Neto PJ. Modelos de avaliação da estabilidade de fármacos e medicamentos para a indústria farmacêutica. Rev. Ciênc. Farm. Básica Apl. 2009;30(2):129-135.

24. Oliveira MA, Yoshida MI, Gomes ECL. Análise térmica aplicada a fármacos e formulações farmacêuticas na indústria farmacêutica. Quim Nova. 2011;34(7):1224-1230.

25. Simon $P$, Veverka $M$, Okuliar J. New screening method for the determination of stability of pharmaceuticals. Int J Pharm. 2004;270(1-2):21-26. 


\section{(i) Revista Eletrônica de Farmácia}

MEIRELLES, L.M.A.

REF-ISSN1808-0804 Vol.XI (4),06-26, 2014.

26. Rodante F, Vecchio S, Catalani G, Tomassetti M. Compatibility between active components of a commercial drug. Farmaco Sci. 2002;57(10):833-843.

27. Júlio TA, Zâmara IF, Garcia JS, Trevisan MG. Compatibility of sildenafil citrate and pharmaceutical excipientes by thermal analysis and LC-UV. J Therm Anal Calorim. 2013;111:2037-2044.

28. Mahlin D, Bergström CA. Early drug development predictions of glass-forming ability and physical stability of drugs. Eur J Pharm Sci. 2013;49:323-332.

29. Chaves LL, Rolim LA, Gonçalves MLCM, Vieira ACC, Alves LDS, Soares MFR et al. Study of stability and drug-excipient compatibility of diethylcarbamazine citrate. J Therm Anal Calorim. 2013;111:2179-2186.

30. Liltorp K, Larsen TG, Willumsen B, Holm R. Solid state compatibility studies with tablet excipients using non thermal methods. J Pharm Biomed Anal. $2011 ; 55(3): 424-428$.

31. Monajjemzadeh F, Hassanzadeh D, Valizadeh H, Siahi-shadbad MR, Mojarrad JS, Robertson TA et al. Compatibility studies of acyclovir and lactose in physical mixtures and commercial tablets. Eur J Pharm Biopharm. 2009;73(3):404-413.

32. Costa SPM, da Silva KER, de Medeiros GCR, Rolim LA, de Oliveira JF, de Lima MCA et al. Thermal behavior and compatibility analysis of the new chemical entity LPSF/FZ4. Thermochim Act. 2013;562:29- 34.

33. Ceschel GC, Badiello R, Ronchi C, Maffei P. Degradation of components in drug formulations: a comparison between HPLC and DSC methods. J Pharm Biomed Anal, 2003; 32: 1067-1072. 


\section{jevista Eletrônica}

MEIRELLES, L.M.A.

REF-ISSN1808-0804 Vol.XI (4),06-26, 2014.

34. Selzer T, Radau M, Kreuter J. The use of isothermal heat conduction microcalorimetry to evaluate drug stability in tablets. Int J Pharm. $1999 ; 184(2): 199-206$.

35. Aulton ME. Delineamento de formas farmacêuticas. 2nd ed. Porto Alegre: Artmed; 2005.

36. Soares MFLA, Soares-Sobrinho JL, da Silva KER, Alves LDS, Lopes PQ, Correia LP et al. Thermal characterization of antimicrobial drug ornidazole and its compatibility in a solid pharmaceutical product. J Therm Anal Calorim. $2011 ; 104: 307-313$.

37. Félix FS, Cides LCS, Angnes L, Matos JR. Thermal behavior study and decomposition kinetics of salbutamol under isothermal and non-isothermal conditions. J Therm Anal Calorim. 2009;95(3):877-880.

38. Huang $Y$, Cheng $Y$, Alexander $K$, Dollimore D. $\underline{A}$ thermal analysis study of methyl salicylate. Thermochim Acta. 2001;367-368:23-28.

39. Rodante F, Vechio S, Catalani G, Tomassetti M. Application of TA and kinetic study to compatibility and stability problems in some commercial drugs. J Therm Anal Calorim. 2001;66(1):155-178.

40. Ozawa T. Thermal analysis: review and prospect. Thermochim Acta. 2000;355: $35-42$.

41. Rodrigues PO, Cardoso TFM, Silva MAS, Matos JR. Aplicação de técnicas termoanalíticas na caracterização, determinação da pureza e cinética de degradação da zidovudina (AZT). Lat Am J Pharm. 2005;24(3):383-387.

42. Duchêne $M$, Jeanmart $L$, Slegers $B$. Stability post-licensure: Compatibility container content - A practical case study. Biologicals. 2009;37(6):407-409. 


\section{i) Revista Eletrônica de Farmácia}

MEIRELLES, L.M.A.

REF-ISSN1808-0804 Vol.XI (4),06-26, 2014.

43. Otsuka M, Ishii M. Improvement of theophylline anhydrate stability at high humidity by surface-physicochemical modification. Colloids Surf B Biointerfaces. $2010 ; 76(1): 158-163$.

44. Tønnesen $\mathrm{HH}$. Formulation and stability testing of photolabile drugs. Int J Pharm. 2001;225(1-2):1-14.

45. Damian F, Fabian J, Friend DR, Kiser PF. Approaches to improve the stability of the antiviral agent UC781 in aqueous solutions. Int J Clin Pharm. 2010;396:1-10.

46. Miller LA, Carrier RL, Ahmed I. Practical considerations in development of solid dosage forms that contain cyclodextrin. J Pharm Sci. 2007;96(7):1691-1707.

47. Ramírez-Rigo MV, Olivera ME, Rubio M, Manzo RH. Enhanced intestinal permeability and oral bioavailability of enalapril maleate upon complexation with the cationic polymethacrylate Eudragit E100. Eur J Pharm Sci. 2014;55:1-11

48. Lopes CM, Lobo JMS, Costa P. Formas farmacêuticas de liberação modificada: polímeros hidrofílicos. Brazilian Journal of Pharmaceutical Sciences. 2005;41(2):143-154.

49. Zhu Y. Properties of polymeric drug delivery systems prepared by hot-melt extrusion. [thesis] Austin: Faculty of the Graduate School of the University of Texas; 2002. 